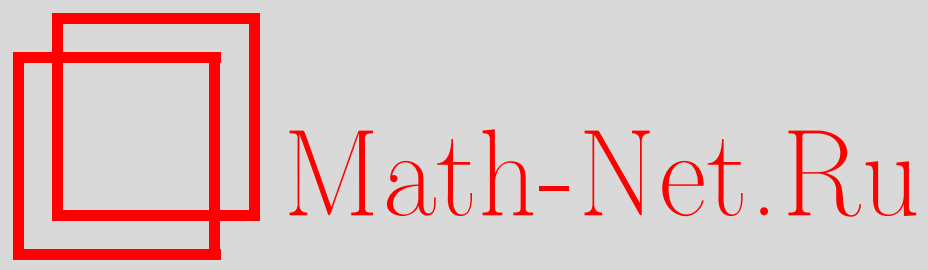

В. И. Бахтин, Усреднение вдоль цепи Маркова, Функи. анализ и его прил., 1996, том 30, выпуск $1,54-56$

DOI: https://doi.org/10.4213/faa504

Использование Общероссийского математического портала MathNet.Ru подразумевает, что вы прочитали и согласны с пользовательским соглашением

http://www . mathnet.ru/rus/agreement

Параметры загрузки:

IP : 54.147 .182 .235

26 апреля 2023 г., 15:04:24

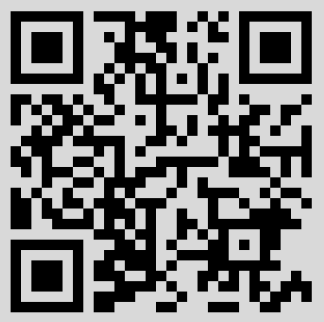




\title{
Усреднение вдоль цепи Маркова
}

\author{
(c) 1996. В. И. БАхтин
}

Рассмотрим стохастический каскад с быстрыми и медленными движениями, определяемый системой

$$
\left\{\begin{array}{l}
x_{i+1}=X_{\varepsilon}\left(x_{i}, y_{i}\right), \quad X_{\varepsilon}(x, y)=x+\varepsilon v(x, y), \\
\mathbf{P}\left(y_{i+1} \in M \mid x_{i}, y_{i}\right)=p\left(x_{i}, y_{i}, M\right) .
\end{array}\right.
$$

Здесь буква $x$ (с индексом или без него) обозначает точку из $\mathbb{R}^{r}$, а $y$ - точку из измеримого пространства $(Y, \mathfrak{A}), \varepsilon$ - малый положительный параметр, $v(x, y)$ - гладкое векторное поле на $\mathbb{R}^{r}$, измеримо зависящее от параметра $y$, и $p(x, y, M)$ - переходная вероятность на $(Y, \mathfrak{A})$ по отношению к аргументам $y \in Y, M \in \mathfrak{A}$, гладко зависящая от параметра $x$. При $\varepsilon=0$, очевидно, каскад вырождается в семейство цепей Маркова, зависящее от параметра $x$.

В теории усреднения изучается отклонение медленной компоненты траектории каскада $\left\{x_{i}\right\}$ от траектории некоторого усредненного каскада на $x$-пространстве в течение времени порядка $\varepsilon^{-1}$. Например, в [1] и [2] для систем, аналогичных (1) (но только с непрерывным временем), установлено, что распределение медленной компоненты удовлетворяет центральной предельной теореме, а также доказаны грубые (с точностью до логарифмической эквивалентности) оценки вероятностей больших уклонений.

В данной же заметке даются точные асимптотики вероятностей больших уклонений (крамеровского типа), в том числе уточняется скорость сходимости в центральной предельной теореме.

Определим оператор $A$ формулой

$$
(A f)(x, y)=\int_{Y} f\left(x, y^{\prime}\right) p\left(x, y, d y^{\prime}\right) .
$$

Можно считать, что он действует в пространстве ограниченных $\mathfrak{B} \times \mathfrak{A}$-измеримых функций (где $\mathfrak{B}$ - борелевская $\sigma$-алгебра на $\mathbb{R}^{r}$ ).

Назовем переходную вероятность равномерно эргодической, если при некотором положительном $\Lambda<1$ для любой ограниченной измеримой функции $f(x, y)$ при всех $x$

$$
\sup _{y}(A f)(x, y)-\inf _{y}(A f)(x, y) \leqslant \Lambda\left(\sup _{y} f(x, y)-\inf _{y} f(x, y)\right) .
$$

В этом случае существует предельный оператор

$$
\bar{A}=\lim _{n \rightarrow \infty} A^{n}
$$

(предел берется в равномерной операторной норме). Очевидно, что функция $\bar{A} f$ не зависит от $y$, а только от $x$. Она является средним значением $f$ по отношению к единственной стационарной мере марковской цепи $(Y, \mathfrak{A}, p(x, y, M))$. 
Усредненным каскадом назовем каскад

$$
\bar{x}_{i+1}=\bar{X}_{\varepsilon}\left(\bar{x}_{i}\right), \quad \text { где } \bar{X}_{\varepsilon}(x)=x+\varepsilon \bar{v}(x), \bar{v}(x)=\bar{A} v(x, y)
$$

(здесь $v$ - векторное поле из системы (1)).

Определим функциональное пространство $B^{n}$ как множество (комплекснозначных) функций $f(x, y)$, которые $n$ раз непрерывно дифференцируемы по $x$, причем все их частные производные измеримы и равномерно ограничены по совокупности переменных. Равномерную $C^{n}$-норму на этом пространстве будем обозначать $\|\cdot\|_{n}$. Ниже всюду будем предполагать, что каждая компонента векторного поля $v$ принадлежит $B^{n}(n \geqslant 1)$, а переходная вероятность $p(x, y, M)$ равномерно эргодична, при каждом фиксированном $M \in \mathfrak{A}$ является элементом пространства $B^{n}$ и каждая ее частная производная по $x$ по отношению к аргументу $M$ является счетно-аддитивным зарядом с равномерно (по $x, y$ ) ограниченной полной вариацией.

Для любой ограниченной последовательности функций $f_{k} \in B^{n}$ введем обозначение $\Delta=\sup \left\|f_{k}\right\|_{n}$ и определим случайные величины

$$
S_{k}=f_{k}\left(x_{0}, y_{0}\right)+f_{k-1}\left(x_{1}, y_{1}\right)+\cdots+f_{1}\left(x_{k-1}, y_{k-1}\right) .
$$

Например, если $f_{i}=v$, то $S_{k}=\varepsilon^{-1}\left(x_{k}-x_{0}\right)$. Если же положить $x_{0}=\bar{x}_{0}$ и

$$
f_{i}(x, y)=\varepsilon^{-1}\left(\left(\bar{X}_{\varepsilon}\right)^{i-1} \circ X_{\varepsilon}(x, y)-\left(\bar{X}_{\varepsilon}\right)^{i}(x)\right),
$$

то, как нетрудно видеть, $S_{k}=\varepsilon^{-1}\left(x_{k}-\bar{x}_{k}\right)$.

ТЕОРема 1. Существуют настолько большое $C$ и малое положительное $R$, что для любой ограниченной последовательности $f_{k} \in B^{n}$ найдутся функции $G_{k}(x, y, \lambda)=G_{k}(\lambda) \in B^{0}$, аналитически зависячие от комплексного параметра $\lambda$ в круге $|\lambda|<R / \Delta$, а также функции

$$
F_{k}(\lambda)=F_{k}(x, y, \lambda)=\lambda F_{k 1}(x, y)+\cdots+\lambda^{n} F_{k n}(x, y), \quad F_{k i} \in B^{n+1-i},
$$

maкие, что при всех $k \leqslant \varepsilon^{-1}$

$$
\begin{gathered}
\mathbf{M}\left\{\exp \left[\lambda S_{k}+\lambda^{n+1} \sum_{j=1}^{k} G_{j}\left(x_{k-j}, y_{k-j}, \lambda\right)\right] \mid x_{0}, y_{0}\right\}=\exp F_{k}\left(x_{0}, y_{0}, \lambda\right) \\
\left\|F_{k i}\right\|_{n+1-i} \leqslant C k \Delta^{i}, \quad\left\|G_{j}(\lambda)\right\|_{0} \leqslant C \Delta^{n+1} \quad(|\lambda|<R / \Delta) .
\end{gathered}
$$

Величины $F_{k i} \cdot i$ ! являются семиинвариантами случайной величины $S_{k}$. При этом $F_{k 1}$ - это математическое ожидание, а $2 F_{k 2}$ - дисперсия $S_{k}$. В случае вещественнозначных функций $f_{k}$ все семиинварианты тоже вещественны. Далее, пусть

$$
\Gamma_{k}(\lambda)=F_{k}(\lambda)-\lambda F_{k 1}, \quad \Psi_{k}(\chi)=\mathbf{P}\left(S_{k}-\mathbf{M} S_{k}<\chi\right), \quad \Phi(\chi)=\frac{1}{\sqrt{2 \pi}} \int_{-\infty}^{\chi} e^{-t^{2} / 2} d t
$$


Теорема 2 (крамеровская асимптотика). Если в условиях теоремы 1 функиии $f_{k}$ вещественнь, $\chi=\Gamma_{k}^{\prime}(\lambda), \lambda \in(0, R / 2)$, то

$$
\begin{aligned}
1-\Psi_{k}(\chi)=( & \left.-\Phi\left(\frac{\chi}{\sqrt{2 F_{k 2}}}\right)\right) \exp \left(\Gamma_{k}(\lambda)-\lambda \Gamma_{k}^{\prime}(\lambda)+\frac{\left(\Gamma_{k}^{\prime}(\lambda)\right)^{2}}{4 F_{k 2}}\right) \\
& \times\left(1+O\left(k \lambda^{m+1}\right)+O\left(\frac{k \lambda}{F_{k 2}}\right)+O\left(k \sqrt{F_{k 2}} \lambda^{m+2}\right)+O\left(k F_{k 2}^{-3 / 2}\right)\right) .
\end{aligned}
$$

Здесь $|O(\xi)| \leqslant \Theta|\xi|$ при всех $|\xi|<\theta$, а положительнье числа $\Theta$ и $\theta$ зависят только от констант $C, \Delta u R$ из оченок (3).

Все $F_{k i}$ являются конструктивно вычисляемыми функциями от начального условия $\left(x_{0}, y_{0}\right)$. В первом приближении значения $F_{k 1}$ и $F_{k 2}$ могут быть найдены следующим образом. Пусть $\bar{x}_{0}, \bar{x}_{1}, \bar{x}_{2}, \ldots$ - траектория усредненного каскада,

$$
\begin{gathered}
\bar{f}_{k}=\bar{A} f_{k}, \quad \tilde{f}_{k}=f_{k}-\bar{f}_{k}, \quad \bar{F}_{k}\left(\bar{x}_{0}\right)=\bar{f}_{k}\left(\bar{x}_{0}\right)+\bar{f}_{k-1}\left(\bar{x}_{1}\right)+\cdots+\bar{f}_{1}\left(\bar{x}_{k-1}\right), \\
\tilde{v}=v-\bar{v}, \quad \tilde{V}=\tilde{v}+A \tilde{v}+A^{2} \tilde{v}+\ldots, \\
\widetilde{F}_{k}=\tilde{f}_{k}+A \tilde{f}_{k-1}+\cdots+A^{k-1} \tilde{f}_{1}+\varepsilon \frac{d \bar{F}_{k}}{d x} \widetilde{V} .
\end{gathered}
$$

Теорема 3. В условиях теоремы 1 при $n \geqslant 3$

$$
\left\|F_{k 1}-\bar{F}_{k}\right\|_{n-2} \leqslant C \Delta, \quad k \leqslant \varepsilon^{-1} .
$$

Если же $n \geqslant 4$, то при всех $k \leqslant \varepsilon^{-1}$

$$
\begin{gathered}
2 F_{k 2}\left(x_{0}, y_{0}\right)=\sum_{j=1}^{k-1} \bar{A}\left[\left(\tilde{f}_{j}+\varepsilon \frac{d \bar{F}_{j}}{d x} \tilde{v}\right)^{2}+2\left(\tilde{f}_{j}+\varepsilon \frac{d \bar{F}_{j}}{d x} \tilde{v}\right) A \widetilde{F}_{j-1}\right]\left(\bar{x}_{k-1-j}\right) \\
+\gamma_{k}\left(x_{0}, y_{0}\right), \quad\left\|\gamma_{k}\right\|_{n-3} \leqslant C \Delta^{2}
\end{gathered}
$$

\section{ЛитературА}

1. Вентиель А. Д., Фрейдлин М. И. Флуктуации в динамических системах под действием малых случайных возмущений. Наука, М. (1979). 2. Вентиель А. Д. Предельные теоремы о больших уклонениях для марковских случайных процессов. Наука, М. (1986).

Белгосуниверситет, физический факультет
Поступило в редакцию 20 мая 1993 г. В переработанном виде 28 сентября 1994 г. 